\title{
The role of Journal of Anesthesia as a flagship anesthesia journal in Asia
}

\author{
Norifumi Kuratani ${ }^{1}$
}

Received: 8 August 2017 / Accepted: 23 September 2017 / Published online: 16 October 2017

(C) Japanese Society of Anesthesiologists 2017

To the Editor:

Academic output, typically measured according to the number of articles published, is insufficient in low and lower middle income countries (LLMICs). As the global standards required for clinical trials have become progressively stricter year by year, the odds that an article from an LLMIC will be accepted in a high-impact indexed journal have decreased. A substantial proportion of LLMICs struggle with severe limitations in research infrastructure and funding. Declining numbers of academic publications and ignorance of global debate regarding the progress of medicine may limit the availability of evidence-based information that could help solve specific nations' health problems.

The lower proportion of articles from LLMICs in indexed journals cannot be attributed solely to lower quality of the submitted articles. Another possible reason is editorial bias against LLMIC authors by journal editors, editorial boards, and publishers from high-income countries [1]. As the official language of major journals is English, there is a significant language barrier for some LLMIC researchers whose primary language is not English. Poorly edited manuscripts are hardly likely to be accepted in high-impact journals, and professional English editing services may not be affordable for LLMIC researchers.

The Journal of Anesthesia (JA) is an official journal of the Japanese Society of Anesthesiologists. JA was launched in 1987 in order to provide a forum where new trends in

Norifumi Kuratani

nori-kuratani@umin.ac.jp

1 Department of Anesthesia, Saitama Children's Medical Center, 1-2 Shin-Toshin, Chuo-ku, Saitama, Saitama 330-8777, Japan
Japanese practice and research are presented [2]. Prof. Amaha, the founding editor-in-chief of JA, intended that Japanese techniques, clinical problems, and ongoing research would become available to anesthesiologists throughout the world, fostering a greater exchange of ideas [2]. Thirty years after the first volume of JA was released, it is clear that JA's original purpose has been achieved with great success. JA is indexed in several medical search engines, including PubMed and Scopus, and is circulated in the anesthesia community all over the globe. JA has served as a catalyst to inspire further development in our field and has brought great benefits to every aspect of anesthesiology in Japan [2].

The rest of Asia, meanwhile, is a mixed region in terms of socioeconomic development. Whereas China and Korea are increasing their presence in anesthesia research [3, 4], many anesthesia societies in LLMICs still have a low scholarly output. Anesthesiologists in these LLMICs would likely find it difficult to launch and maintain an international journal comparable to JA that would meet the global standards of medical research due to the shortage of funds and the lack of clear individual-level benefits resulting from such an undertaking. As a result, the majority of research findings from LLMICs are not indexed in major medical databases and might in fact be inaccessible to the rest of the world.

JA plays a role in counteracting this tendency by publishing substantial quantities of academic output from other Asian countries. In 2016, JA published 85 original articles, of which 29 were from Asian countries other than Japan (Fig. 1). The Asian articles distributed by JA made a substantial impact on the progress of anesthesia research. For example, that by Zhang et al., from China, reported the results of a meta-analysis regarding an association between childhood exposure to single general anesthesia and later neurodevelopmental outcome [5]. The authors summarized the currently available evidence regarding anesthesia 
Fig. 1 The geographic distribution of original articles published in Journal of Anesthesia, volume 30 (2016). $n$ numbers of articles

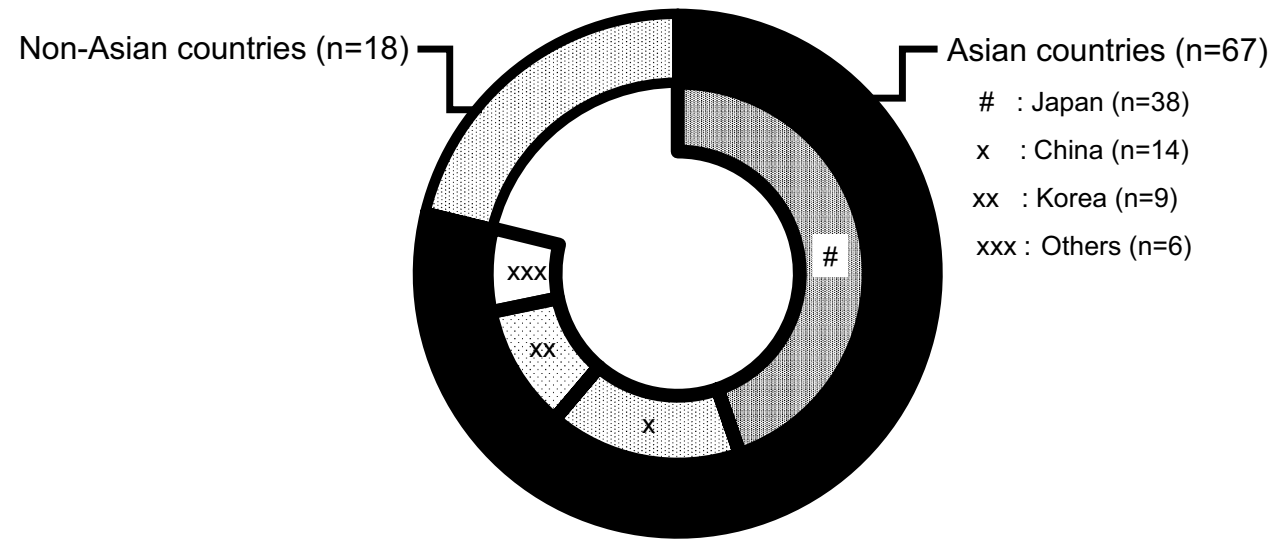

4. Hirota K. Anesthesia research in Japan: seeds of recovery and the role of the Journal of Anesthesia. J Anesth. 2017. doi:10.1007/ s00540-017-2377-4.

5. Zhang H, Du L, Du Z, Jiang H, Han D, Li Q. Association between childhood exposure to single general anesthesia and neurodevelopment: a systematic review and meta-analysis of cohort study. J Anesth. 2015;29:749-57.

6. Erdil F, Begeç Z, Kayhan GE, Yoloğlu S, Ersoy MÖ, Durmuş M. Effects of sevoflurane or ketamine on the QTc interval during electroconvulsive therapy. J Anesth. 2015;29:180-5.

7. Srivastava VK, Agrawal S, Kadiyala VN, Ahmed M, Sharma S, Kumar R. The efficacy of pregabalin for prevention of catheterrelated bladder discomfort: a prospective, randomized, placebocontrolled double-blind study. J Anesth. 2015;29:212-6.

8. Karaman Y, Abud B, Tekgul ZT, Cakmak M, Yildiz M, Gonullu M. Effects of dexmedetomidine and propofol on sedation in patients after coronary artery bypass graft surgery in a fast-track recovery room setting. J Anesth. 2015;29:522-9.

9. Sun B, Wang J, Bo L, Zang Y, Gu H, Li J, Qian B. Effects of volatile vs. propofol-based intravenous anesthetics on the alveolar inflammatory responses to one-lung ventilation: a meta-analysis of randomized controlled trials. J Anesth. 2015;29:570-9.

10. Arslan ZI, Alparslan V, Ozdal P, Toker K, Solak M. Face-toface tracheal intubation in adult patients: a comparison of the Airtraq $^{\mathrm{TM}}$, Glidescope ${ }^{\mathrm{TM}}$ and Fastrach ${ }^{\mathrm{TM}}$ devices. J Anesth. 2015;29:893-8. 